\title{
Self-assembly of biaxial ordering and molecular tilt angle of chiral smectic liquid crystals in homeotropically aligned cells investigated using infrared spectroscopy
}

\author{
R. Korlacki, ${ }^{1, *}$ A. Fukuda, ${ }^{1}$ J. K. Vij,,${ }^{1, \dagger}$ A. Kocot, ${ }^{1,2}$ V. Görtz, ${ }^{3}$ M. Hird, ${ }^{3, \ddagger}$ and J. W. Goodby ${ }^{3}$ \\ ${ }^{1}$ Department of Electronic and Electrical Engineering, Trinity College, University of Dublin, Dublin 2, Ireland \\ ${ }^{2}$ Institute of Physics, University of Silesia, 40-007 Katowice, Poland \\ ${ }^{3}$ Department of Chemistry, University of York, York Y010 5DD, United Kingdom
}

(Received 11 March 2005; revised manuscript received 21 April 2005; published 12 October 2005)

\begin{abstract}
Temperature dependences of the infrared absorbance have been measured for the four chiral liquid crystal samples in the homeotropic cell configuration. It is shown that the values of the orientational order parameter $S$ obtained using this method exhibit a remarkable similarity to the x-ray diffraction results of the smectic layer spacing and lead to accurate values of the molecular tilt angle. This has important consequences for the existing interpretation of the $\mathrm{x}$-ray data. The proposed method, in many cases, may be considered as a valuable alternative to the $\mathrm{x}$-ray diffraction, giving additional, important information about the orientations and the ordering of the molecular fragments. It is found that if the experimentally obtained order parameter is low, then the molecular biaxiality is exceptionally large. The average phenyl ring plane is found to lie close to the molecular tilt plane.
\end{abstract}

DOI: 10.1103/PhysRevE.72.041704

PACS number(s): 61.30.Gd, 42.70.Df, 64.70.Md, 78.30.Jw

\section{INTRODUCTION}

Since the beginning of 1990s, the infrared spectroscopy has been found to be an extremely powerful method for the investigation of ferroelectric liquid crystals at a microscopic level. After a preliminary paper on the subject by Zgonik et al. [1], Kocot et al. [2] successfully employed the infrared spectroscopy for determining the tilt angle and the order parameter. This was soon followed by Kim et al. [3] who used this technique for proving the rotational bias in ferroelectric and antiferroelectric liquid crystals. Since these earlier works, the infrared spectroscopy has widely been used to investigate an orientational order in liquid crystals. These works were reviewed by Kocot et al. [4]. Due to a progress in the computational methods, a series of papers have recently appeared in the literature [5-13], where infrared spectroscopy has significantly been supported by ab initio calculations. This promises to be a fruitful way for the future. However, in most cases, liquid crystals have been studied using this technique in planar homogeneous alignments only. Investigating of liquid crystalline cells aligned homeotropically may yield additional important information. In this paper we show that in such experiments, the temperature dependence of the orientational order parameter obtained from the infrared absorbance is a close analogy to the $\mathrm{x}$-ray results of smectic layer spacing. Moreover, from the same data we are able to obtain reasonably precise values of the molecular tilt angle and order parameters and in addition observe effects of the orientation and the rotational bias of the molecu-

\footnotetext{
*Current address: Institute of Physical and Theoretical Chemistry, University of Tübingen, 72076 Tübingen, Germany.

${ }^{\dagger}$ Author to whom correspondence should be addressed. Email address: jvij@tcd.ie

${ }^{\ddagger}$ Permanent address: Department of Chemistry, University of Hull, Hull, UK.
}

lar short axes. This offers significant advantages over the $\mathrm{x}$-ray diffraction.

The homeotropic orientation means that the IR beam is parallel to the smectic layer normal (Fig. 1). The measured absorbance, in the laboratory reference system, therefore, is (according to Fig. 1 and after normalizing with respect to the isotropic phase $[12,14,15])$

$$
A=\left(A_{X}+A_{Y}\right) / 2 A_{0}=1-S P_{2}(\cos \beta)+\frac{1}{2} D \sin ^{2} \beta \cos 2 \gamma,
$$

where $\beta$ and $\gamma$ are the Euler angles describing the orientation of the transition dipole moment in the molecular reference system; $P_{2}(x)=\frac{1}{2}\left(3 x^{2}-1\right)$ is the second Legendre polynomial of $x, S$ and $D$ are two, out of a complete set of four, independent order parameters of the second rank tensor as defined by the Saupe ordering matrix

$$
\begin{gathered}
S \equiv S_{z z}^{Z}, D \equiv S_{x x}^{Z}-S_{y y}^{Z}, P \equiv S_{z z}^{X}-S_{z z}^{Y}, \\
C \equiv\left(S_{x x}^{X}-S_{y y}^{X}\right)-\left(S_{x x}^{Y}-S_{y y}^{Y}\right),
\end{gathered}
$$

where

$$
S_{\alpha \beta}^{i}=\left\langle\frac{1}{2}\left(3 l_{i, \alpha} l_{i, \beta}-\delta_{\alpha \beta}\right)\right\rangle, i=X, Y, Z ; \alpha, \beta=x, y, z,
$$

$l_{i, \alpha}$ is the cosine of the angle between the molecular axis $\alpha$ and the laboratory or phase axis $i$. The order parameter $S$ (called the uniaxial or nematic order parameter) describes an alignment of long molecular axes (a probability of finding of the molecular long axis $z$ along the direction $Z$ ). $D$ (the molecular biaxiality parameter) describes an orientational order of short axes and biasing of the rotation about the long molecular axes (a difference between the distribution of molecular axes $x$ and $y$ with respect to the axis $Z$ ). $P$ (the phase biaxiality parameter) describes an average tilting of molecules [15] (a difference in the probabilities of finding the 


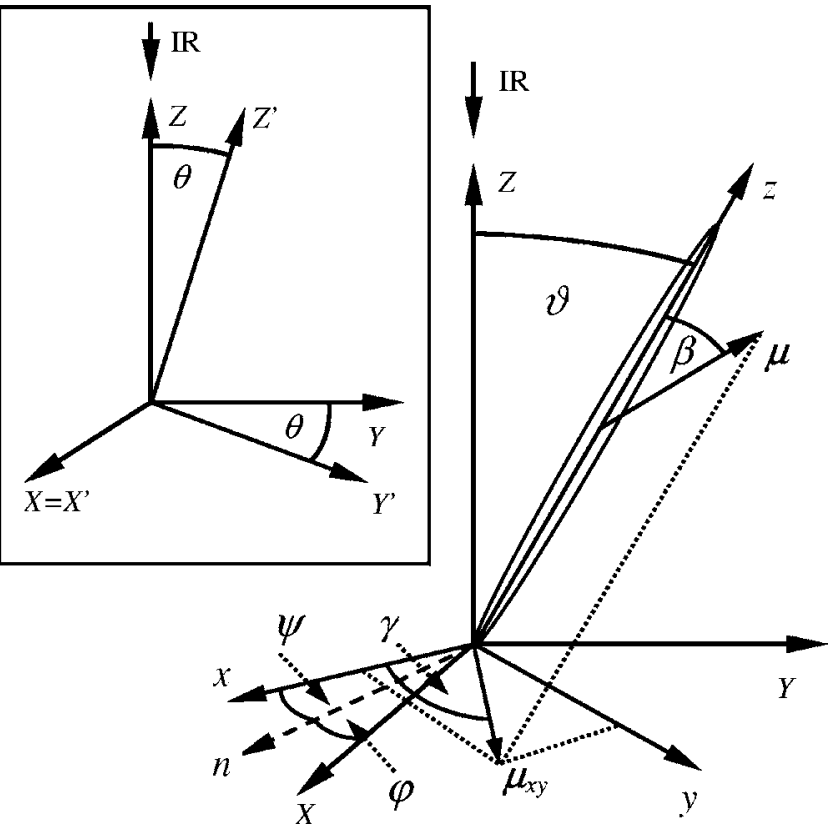

FIG. 1. Schematic diagram of experiments with homeotropically aligned liquid crystals. $X, Y, Z$ denote axes of the laboratory reference system. $x, y, z$ denote axes of the molecular reference system. $\varphi, \vartheta, \psi$ are Euler angles describing an orientation of the molecular reference system vs the laboratory reference system ( $n$ is an axis perpendicular to the $Z z$ plane-the line of nodes, $\varphi$ describes the rotation around $Z, \psi$ describes the rotation around $z$ ). $\beta$ and $\gamma$ describe an orientation of the IR transition dipole moment $\boldsymbol{\mu}$ in the molecular reference system. $\boldsymbol{\mu}_{x y}$ is a projection of the transition dipole moment $\boldsymbol{\mu}$ on the $x y$ plane. In the inset: a definition of the local system discussed in the text; $X^{\prime}, Y^{\prime}, Z^{\prime}$ are axes of the local system; $\theta$ is the (in-layer) director tilt angle, $\theta=\langle\vartheta\rangle$ (the averaging is made locally, i.e., for a single smectic layer).

molecular axis $z$ along the axes $X$ and $Y$ ). $C$ (the mixed biaxiality) describes a coupling between $D$ and $P$.

It is important to emphasize that in the sample aligned homeotropically, there exist all possible orientations of tilting directions around the smectic layer normal (an isotropic distribution of angles $\varphi$, i.e., all values of $\varphi$ are equally probable). This has been confirmed by the absence of IR dichroism, using the polarized IR beam, for all the samples and the phases studied.

Locally, at a level of single smectic layer, the transition from $\operatorname{Sm} A^{*}$ phase to any tilted $\mathrm{Sm} C^{*}$ phase changes a symmetry of the single molecule distribution function, from uniaxial to biaxial. The tilt plane (i.e., the plane defined by the director and the smectic layer normal) is then the only plane of symmetry of the system. For the tilted smectic phases, therefore, it is convenient to use a reference system fixed to the local director, and to the tilt plane, for example by rotating the laboratory reference system around $X$ by angle $\theta$ (see Fig. 1, in the inset). In this reference system, order parameter $P^{\prime}=0$ (because $S_{z z}^{X^{\prime}}=S_{z z}^{Y^{\prime}}$ ), and the order parameter $S^{\prime}$ has the same meaning for nematics and tilted smectics and is often called the molecular order parameter. (Values of the order parameters calculated with respect to arbitrary chosen axes, fixed in space, are affected by the

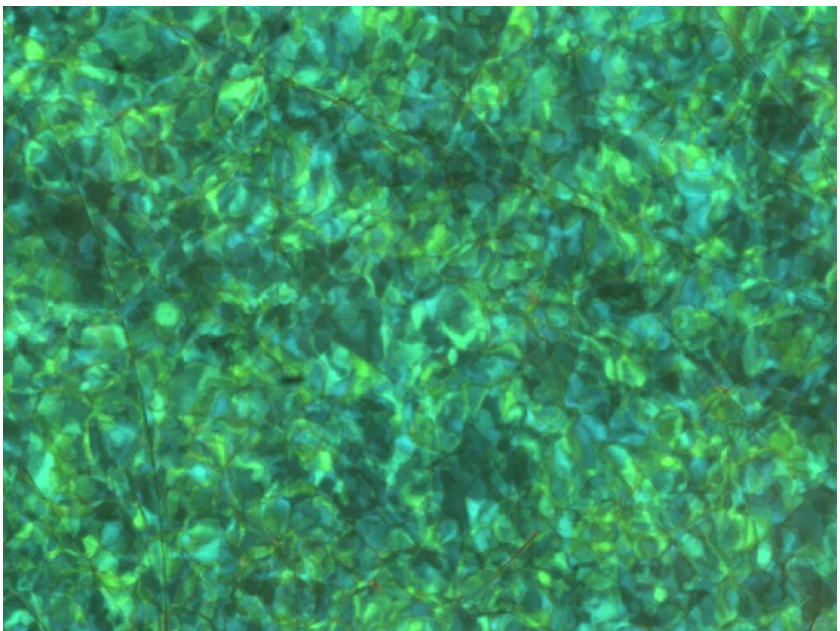

FIG. 2. (Color online) Schlieren texture of 12OF1M7 in $\mathrm{SmC}_{A}^{*}$ phase $\left(T=42{ }^{\circ} \mathrm{C}\right)$. The diameter of the visible area is $<1 \mathrm{~mm}$. The diameter of the IR beam used in the investigations is approximately $6 \mathrm{~mm}$.

appearance of the tilt, and consequently, the order parameters in the laboratory reference system are often called "apparent.") Hence the nonpolarized IR beam is parallel to the smectic layer normal (i.e., is always within the tilt plane and rotated from the local director by $\theta$ ), each smectic layer and each randomly oriented, microscopic domain (see Fig. 2) give exactly the same contribution to the macroscopic absorbance. Therefore, the experiments using the homeotropic cell configuration are independent of the presence of the smectic helical structure. Finally, we can write the formula for the absorbance in the local reference system as [12]

$$
A=\frac{1}{2}\left[A_{Z} \sin ^{2} \theta+A_{Y} \cos ^{2} \theta+A_{X}\right],
$$

which leads to the following final equation for the absorbance:

$$
\begin{aligned}
A= & 1-S^{\prime} P_{2}(\cos \theta) P_{2}(\cos \beta) \\
& +\frac{1}{2}\left[D^{\prime} P_{2}(\cos \theta)-\frac{1}{2} C^{\prime} \sin ^{2} \theta\right] \sin ^{2} \beta \cos 2 \gamma .
\end{aligned}
$$

Here the symbols $S^{\prime}, D^{\prime}$, and $C^{\prime}$ denote the order parameters in the new reference system.

\section{EXPERIMENT}

\section{A. Materials used for experiments}

Four materials have been chosen for these experiments: two of these are well-known, thoroughly investigated classic liquid crystals: ferroelectric 3M2CPHOB (also known as C6 [17-19]) and antiferroelectric MHPOBC [20]; 12OF1M7 (frequently referred as AS573 [21-23]) and novel 12OBPTFM5O1 synthesized at the University of Hull, UK. Structures and phase sequences of the investigated materials are shown in Fig. 3. The samples 3M2CPHOB and $\mathrm{MH}-$ POBC have been included in the investigation because they 

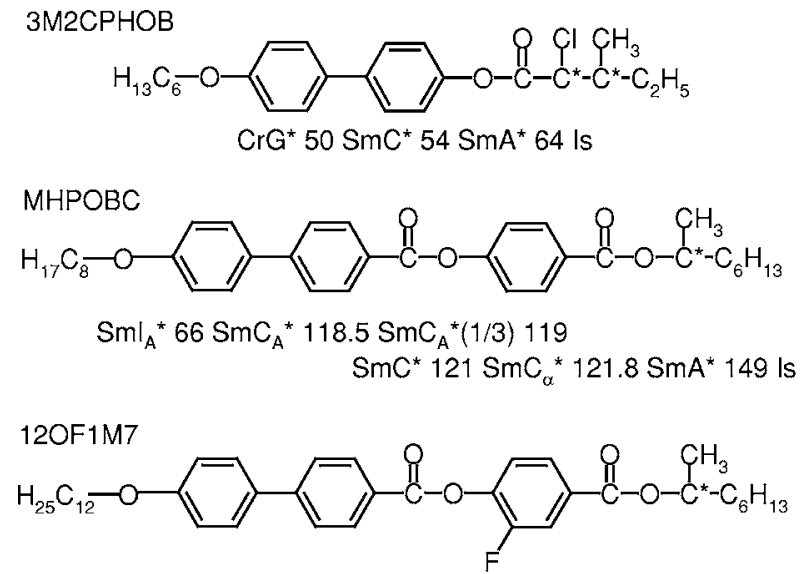

$\mathrm{SmC}_{\mathrm{A}}{ }^{*} 77 \mathrm{SmC}_{\mathrm{A}}{ }^{*}(1 / 3) 80.5 \mathrm{SmC}_{\mathrm{A}}{ }^{*}(1 / 2) 85.5$ $\mathrm{SmC}^{*} 93 \mathrm{SmC}_{\alpha}^{*} 94.5 \mathrm{SmA}^{*} 106 \mathrm{ls}$

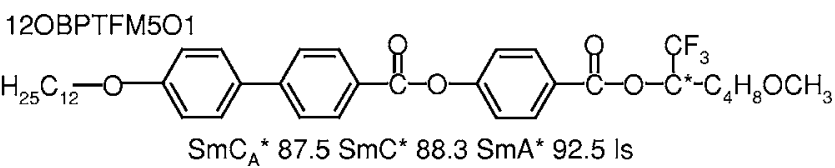

FIG. 3. Structures of the samples investigated and their phase sequences; ferrielectric subphases are labeled as $\operatorname{SmC}_{A}^{*}\left(q_{T}\right)$ [16], where $q_{T}=1 / 3$ and $1 / 2$ denote three-layer and four-layer phases, respectively; all temperatures are in ${ }^{\circ} \mathrm{C}$. Please note that because the results of all experiments shown in this paper have been obtained using thin cells, the temperatures of the phase transitions may be shifted with respect to those given above.

may be considered as reference compounds, on which works are widely published in the literature. Two compounds in the set (MHPOBC and 12OF1M7) exhibit second order transition between $\operatorname{Sm} A^{*}$ and $\mathrm{SmC}^{*}$ phases and a rich variety of $\mathrm{Sm}_{A}^{*}\left(q_{T}\right)$ subphases. In 3M2CPHOB and 12OBPTFM5O1, the $\mathrm{Sm} A^{*}-\mathrm{Sm} C^{*}$ transition is of the first order and the subphases are suppressed. Such a selection gives the best overview of the results obtained.

\section{B. Sample preparation and measurements}

Samples for the experiments were sandwiched between two $\mathrm{ZnSe}$ windows covered with orienting layers of carboxylatochromium complexes (chromolane) in order to obtain the homeotropic alignment. Mylar film of thickness of $5 \mu \mathrm{m}$ has been used as spacers. The cells were filled at a temperature a few degrees above the transition to the isotropic phase. All experiments were carried out using Bio-Rad FTS-6000 Fourier transform infrared spectrometer with an accumulation over 32 scans, with a temperature step of $0.3{ }^{\circ} \mathrm{C}$. The temperature was stabilized during $300 \mathrm{~s}$ for each point using a homemade controller with an accuracy $<0.05^{\circ} \mathrm{C}$. Infrared bands chosen for further considerations were then fitted with a numerical approximation of the Voigt function.

Along with infrared experiments, the molecular structures and their vibrational spectra were calculated using the GAUSSIAN 98W program [24]. The method of the density functional theory (DFT) incorporating the hybrid B3LYP functional and a standard polarized $6-31 G^{*}$ basis set has been chosen for the calculations. For the smallest molecule,
TABLE I. Scaling factors for SQM procedure, X, Y, Z denote heavy (nonhydrogen) atoms; some original factors not applicable for the systems studied have been removed.

\begin{tabular}{lc}
\hline \hline Symmetry of Modes & Scaling Factor \\
\hline X-Y stretching & 0.9254 \\
C-H stretching (aliphatic) & 0.8890 \\
C-H stretching (aromatic) & 0.9150 \\
X-Y-Z bending & 0.9923 \\
C-C-H bending & 0.9473 \\
H-C-H bending & 0.9171 \\
Out of plane & 0.9711 \\
C-O-H bending & 0.9047 \\
Torsions of conjugated systems & 0.9389 \\
Torsions of single-bonded systems & 0.8980 \\
Linear deformations & 0.8905 \\
\hline \hline
\end{tabular}

$3 \mathrm{M} 2 \mathrm{CPHOB}$, of only 59 atoms, the calculations were carried out with higher accuracy using the diffusive $6-31+G^{*}$ basis set (recommended for systems with a significant negative charge; for $3 \mathrm{M} 2 \mathrm{CPHOB}$ the presence of a chlorine atom in the structure is the reason for this approach). Force fields obtained from calculations were scaled using the scaled quantum-mechanical (SQM) method [25,26]. A set of factors for the SQM method is given in Table I $[27,28]$. The above procedure has already been successfully applied to several liquid crystalline systems $[9,10,12,13]$ in the literature.

\section{RESULTS AND DISCUSSION}

\section{A. Infrared spectra of the investigated compounds}

Figure 4 shows a comparison between the experimental and the calculated infrared spectra of the compounds investigated here. Table II contains basic assignments of the most important bands based on the results of the DFT calculations (experimental frequencies for the isotropic phase).

\section{B. Infrared studies as an alternative to $x$-ray diffraction and a method for determining the tilt angle}

For further consideration, a phenyl ring stretching band $\left(1600 \mathrm{~cm}^{-1}\right)$ has been chosen. This band is widely selected for study as it is well defined and its transition dipole moment is almost parallel to the long molecular axis $[3,5,6,11,12,22,23,29-32]$. This arises from the experimental analysis of the IR dichroism and is supported by the DFT calculations. The angle $\beta$ for this transition dipole moment is usually below $10^{\circ}$. However, if we consider the strong bending of chiral tails for antiferroelectric compounds [31], which in reality cannot be properly reproduced in the DFT calculation of a single isolated molecule, it is much more reliable to say that the transition dipole moment of this band is parallel to the main axis of the molecular core. The behavior would be easier to analyze after conversion to an order parameter: from Eq. (1) for any band, where the transition dipole mo- 

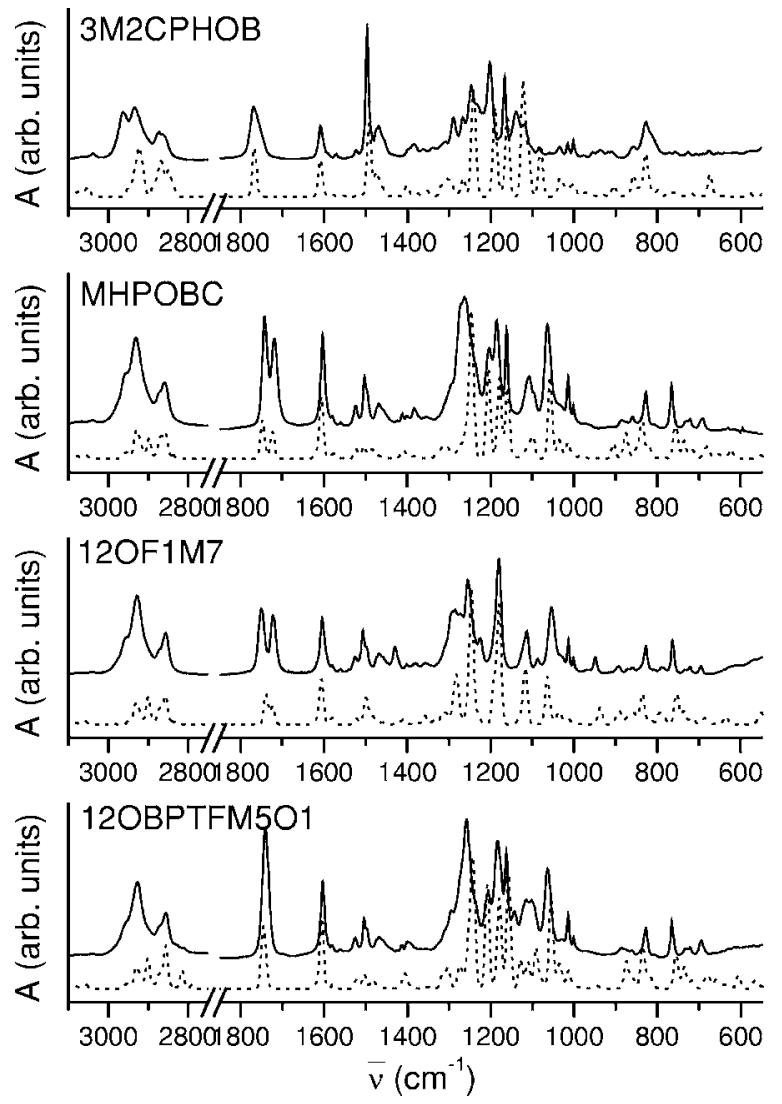

FIG. 4. Infrared spectra of the investigated compounds; solid lines-experimental spectra, dotted lines - calculated spectra. Due to relatively small intensities of bands in lower frequency parts of the calculated spectra, their vertical scales below $900 \mathrm{~cm}^{-1}$ have been expanded. All the experimental spectra have been collected a few degrees above a transition to the isotropic phase for each compound.

ment is parallel to the long molecular axis, the formula can be simplified (because $\beta \approx 0$ ) to

$$
S=1-A .
$$

Figure 5 shows temperature dependence of the apparent order parameter $S$ for the various compounds. The temperature dependence is quite similar for all the compounds studied: strong jump in the value at the transition from isotropic to $\mathrm{Sm} A^{*}$ phase, from 0 to $0.6-0.8$, followed by a slight increase, obviously connected with a decrease in the thermal fluctuations. At the transition to the $\mathrm{Sm} C^{*}$ phase, however, an effect of the appearance of the tilt is reflected in a decrease in the value of the order parameter. The dependence of the apparent order parameter for MHPOBC is surprisingly similar to the behavior of smectic layer spacing for this material as published by Kim et al. [32]. Figure 6 shows the region corresponding to that given in Ref. [32], which is shown in the inset.

An increase in the smectic layer thickness near the $\mathrm{SmC}_{A}^{*}$-SmI $I_{A}^{*}$ transition was already observed in several antiferroelectric compounds [33,34]. An explanation of this effect given by Takanishi et al. [34] in terms of a decrease in an interdigitation with a reduction of temperature is not satisfactory and needs to be reconsidered. This problem is under further investigation in our laboratory and will be described in detail separately. However, the variations of the absorbance, near the transition $\mathrm{SmC} C^{*}-\mathrm{Sm} A^{*}$, and of the smectic layer spacing are driven by an appearance of the tilt. Therefore, it is possible to determine the tilt angle from our data.

Comparing Eqs. (1) and (4) for the case $\beta \approx 0$ leads to the following result [35]:

$$
S=S^{\prime} P_{2}(\cos \theta),
$$

which allows us to determine $\theta$ if the $S$ and $S^{\prime}$ values are known

$$
\theta=\arccos \sqrt{\frac{2}{3}\left(\frac{S}{S^{\prime}}+\frac{1}{2}\right)} .
$$

The values of the apparent order parameter $S$ are experimentally measured as shown in Fig. 5. We can obtain $S^{\prime}$ by extrapolating $S$ from the $S m A^{*}$ phase, where $S^{\prime}=S$, to lower temperature phases. The fit can be obtained using the power law

$$
S=B\left(T_{c}-T\right)^{c} .
$$

Of course, the above equation does not describe properly the character of the phase transition Is-Sm $A^{*}$, which obviously is a first order transition, but gives a reasonably good agreement with the experimental data in the $\mathrm{Sm} A^{*}$ phase range (Figs. 7 and 8). In Eq. (8), $B, T c$, and $c$ are constants. The fits and further results of the tilt angles are shown for $3 \mathrm{M} 2 \mathrm{CPHOB}$ (Fig. 7), MHPOBC, and 12OF1M7 (Fig. 8) as compounds, for which the corresponding values obtained using $\mathrm{x}$-ray diffraction are known. Due to the first order $\mathrm{Sm} A^{*}-\mathrm{Sm} C^{*}$ transition in 3M2CPHOB, we may expect that the order parameter may exhibit some discontinuity. Indeed

TABLE II. Assignments of the most important infrared bands.

\begin{tabular}{lcccc}
\hline \hline & \multicolumn{4}{c}{ Frequency $\left(\mathrm{cm}^{-1}\right)$} \\
Mode & 3M2CPHOB & MHPOBC & 12OF1M7 & 12OBPTFM5O1 \\
\hline $\mathrm{C}=\mathrm{O}$ stretching (core) & 1769 & 1742 & 1750 & 1740 \\
$\mathrm{C}=\mathrm{O}$ stretching (chiral) & 1719 & 1721 & 1740 \\
Phenyl ring stretching & 1609 & 1603 & 1604 & 1603 \\
Phenyl ring in-plane deformation & 1167 & & 1180 & 766 \\
Phenyl ring out-of-plane deformation & & 765 & 764 & 764 \\
\hline \hline
\end{tabular}



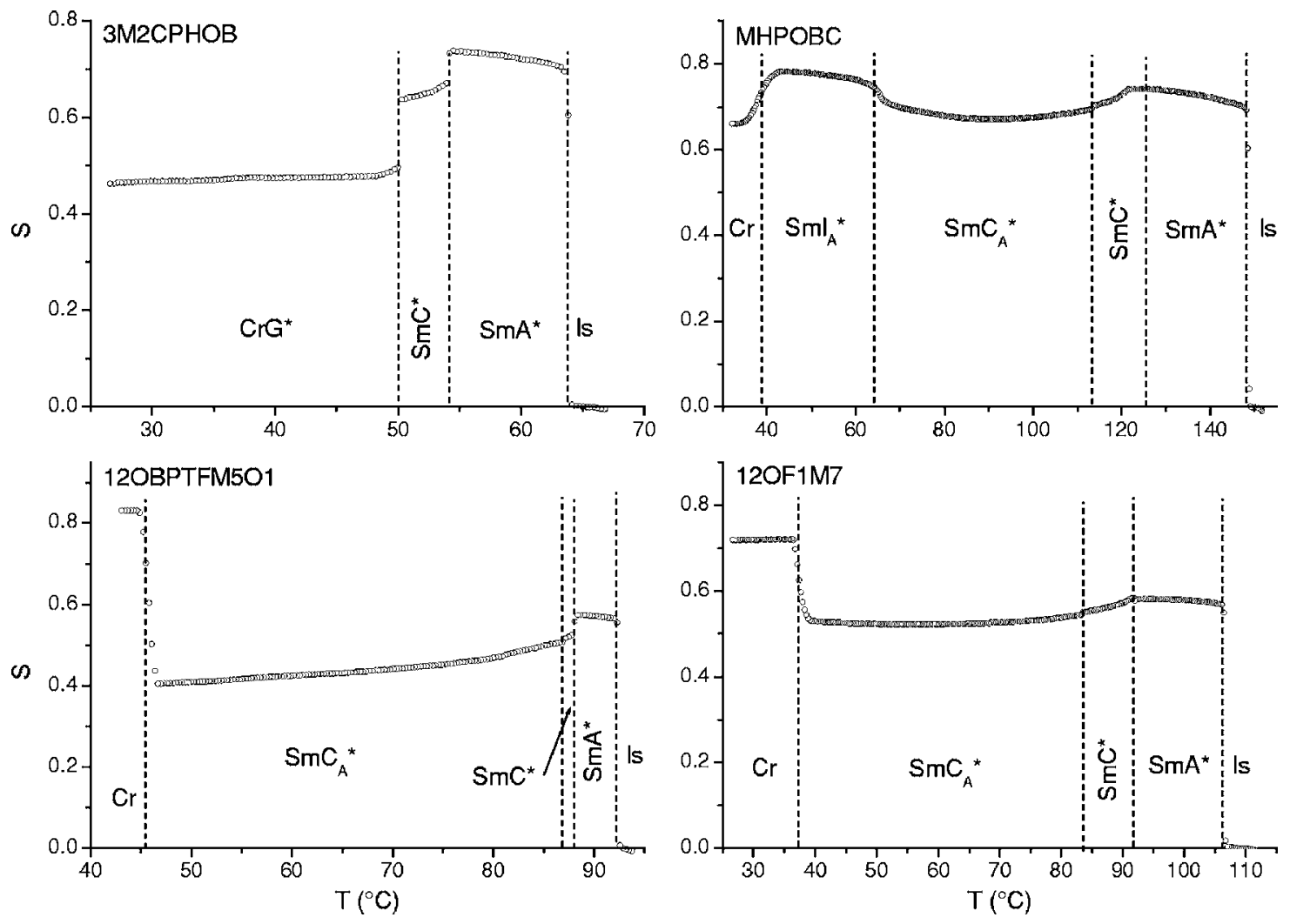

FIG. 5. Order parameter $S$ vs temperature for the investigated compounds; due to convenience, the label $\mathrm{SmC}^{*}$ in the case of MHPOBC and 12OF1M7 includes also the $\operatorname{Sm}_{\alpha}^{*}$ phase and all very narrow $\operatorname{SmC}_{A}^{*}\left(q_{T}\right)$ subphases.

such a discontinuity is visible in the order parameters obtained using NMR $[18,19]$. Although, the values of the order parameter $S$ obtained using both techniques (IR and NMR) are slightly different, nevertheless, we may expect that the discontinuity as an increase of $6 \%$ in $S$ is really the same. The values of the tilt angle obtained with and without the discontinuity being considered are presented in Fig. 7(b). The latter ones are in perfect agreement with those given by

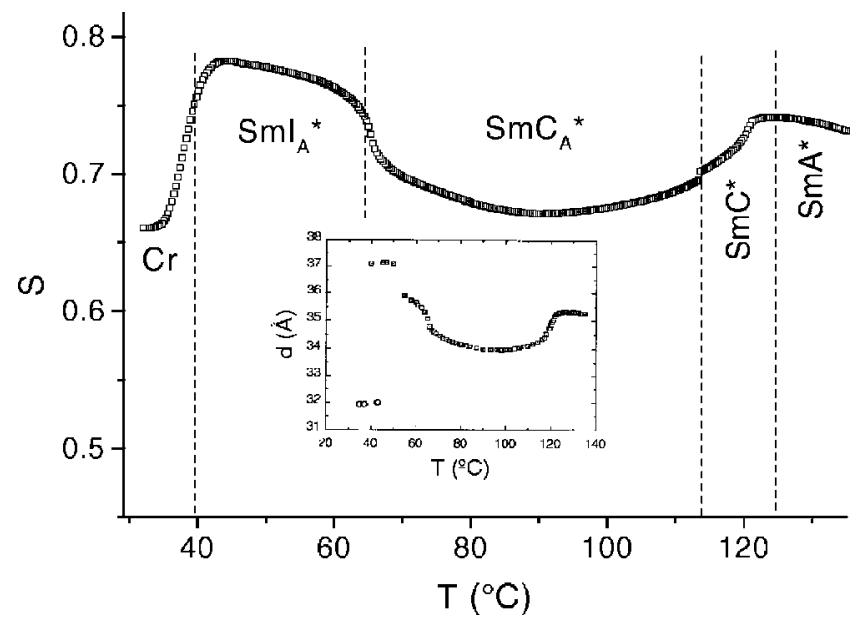

FIG. 6. Order parameter for the MHPOBC. The temperature dependence of the smectic layer spacing is given in the inset (reprinted from Ref. [32]). The region labeled $\mathrm{SmC}^{*}$ includes $\mathrm{Sm}^{*}$ phase, $\mathrm{Sm}_{\alpha}^{*}$ phase, and the various narrow $\operatorname{SmC}_{A}^{*}\left(q_{T}\right)$ subphases.
Bahr and Heppke [17]. For MHPOBC and 12OF1M7, which exhibit second order transition between $\operatorname{Sm} A^{*}$ and tilted smectic phases, we cannot expect any discontinuity. The calculated tilt angles are given for MHPOBC in Fig. 8(b) and for $12 \mathrm{OF} 1 \mathrm{M} 7$ in Fig. 8(c). It appears that the agreement between the IR and X-ray results is not only qualitative, but also the maximum tilt angles agree reasonably well. The tilt angle calculated from the X-ray data given in Ref. [32] for MHPOBC is approximately $16^{\circ}$, which is exactly the same as that obtained from IR. For $12 \mathrm{OF} 1 \mathrm{M} 7$ the agreement between IR $\left(18.3^{\circ}\right)$ and $\mathrm{x}$-ray $\left(\sim 20^{\circ}\right.$ —as shown by Matkin et al. [36]) is only slightly worse.

The overall similarity of results obtained using both techniques has important consequences. With the $1600 \mathrm{~cm}^{-1}$ band, we can in fact observe (especially by taking into account a distribution of the long molecular axes around the director) a behavior of the main axes of the molecular cores. It means that the molecular cores tilt by exactly the same angle as that deduced from the smectic layer shrinkage. The usual explanation, that the steric tilt angle saturates always at lower values than the optical tilt because the molecular cores tilt more than the molecular tails (see, for example, Mills et al. [37]), seems not to be the case. A detailed discussion of the various possible explanations is given below:

(i) The temperature dependences of the smectic layer spacing obtained using x-ray and the order parameters obtained using IR, leading to a deduction of the tilt angle, have been carried out in the homeotropic geometry, while the optical tilt angle is measured in a planar homogeneous cell. Therefore, one possible explanation is that the molecules be- 


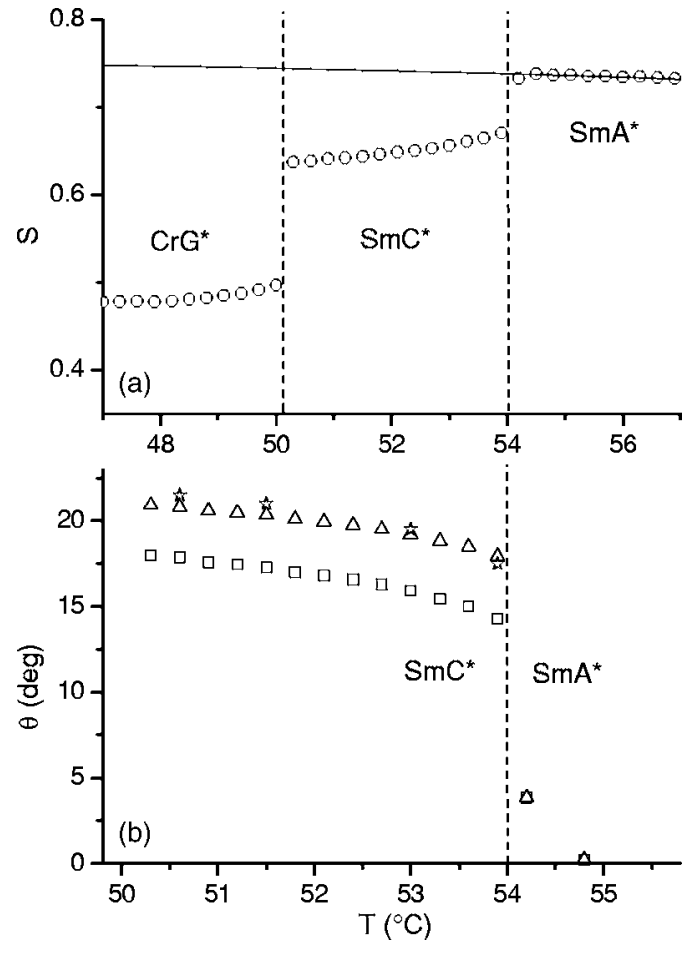

FIG. 7. Fit of the order parameter and its extrapolation to the lower temperatures (a) and tilt angle values (b) for $3 \mathrm{M} 2 \mathrm{CPHOB}$; circles denote the experimental order parameter, the line is drawn from a fit using Eq. (8). Squares - values of the tilt angle obtained [using Eq. (7)] with an assumption of the order parameter varying continuously from $\mathrm{Sm} A^{*}$ to $\mathrm{SmC}^{*}$; triangles-values of the tilt angle obtained by taking into account a jump of the order parameter; stars-x-ray tilt as given by Bahr and Heppke [17].

have differently in both cases, the behavior of the bulk sample (the influence of substrates on molecular behavior in the homeotropic alignment is believed to be insignificant) and the sample in surface-stabilized cells cannot be directly compared to each other. Moreover, the tilt angle obtained from the rotation of the absorbance profile of the same band $1600 \mathrm{~cm}^{-1}$ in homogeneous cells usually give values of the tilt angle much closer to the optical ones (see, for example, Refs. $[6,12,22])$, higher than those obtained from the homeotropic geometry.

(ii) The cores and the entire set of molecules tilt exactly as observed by x-ray and also by IR, while the optical tilt, forced by an external electric field, is partially a result of the coupling between the spontaneous polarization of the material and an external electric field (the electroclinic effect).

It is worth noting that accepting either of the above scenarios implies that liquid crystalline molecules are much more rigid than generally anticipated, the molecules tilt as a whole, and a relation between the molecular cores and the molecular tails does not change significantly in tilted phases. Consequently interpenetration of molecular tails between the layers cannot be extensive. It seems too that a slight increase of smectic layer spacing with decreasing of temperature, as observed within $\mathrm{Sm} A^{*}$ phase for many antiferroelectric liquid crystal (AFLC) compounds (see, for example, Refs. $[32,37])$, can be directly attributed to an increased orientational order.
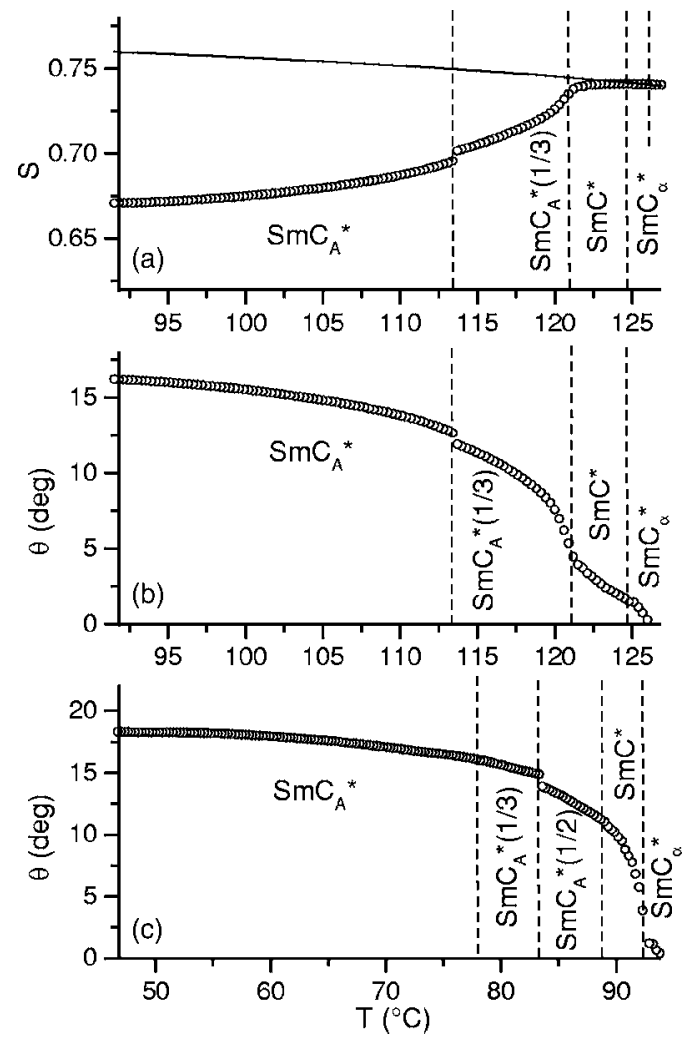

FIG. 8. Fit of the order parameter and its extrapolation to the lower temperatures and tilt angle values [obtained using Eq. (7)] for MHPOBC [(a) and (b)] and 12OF1M7 (c). Due to the continuous transition from $\mathrm{Sm} A^{*}$ phase to tilted smectic phases, the fit has been carried out for points far from the transition temperature. The order parameter in $\mathrm{SmC}_{\alpha}^{*}$ phase due to a small tilt is approximately the same as in $\mathrm{Sm} A^{*}$ phase; however, it enables a calculation of reasonable values of the tilt [please note a nonlinear character of Eq. (7)]. Values of the tilt angle for MHPOBC virtually decrease at temperatures lower than those shown in the figure. This is clearly an effect of the underlying $\operatorname{Sm} I_{A}^{*}$ phase, exactly as shown by Takanishi et al. [34] for a similar compound. Because the origin of this effect is not discussed here, we are showing the values of the tilt angle only to the point of its maximum value.

\section{Orientation of the molecular cores: phenyl ring out-of-plane vibrations}

Some important information about the orientation of the molecular cores can be obtained by analyzing the phenyl ring out-of-plane band $\left(760 \mathrm{~cm}^{-1}\right)$. Such a band could not be observed in the material 3M2CPHOB (there are some out-ofplane vibrations near $800 \mathrm{~cm}^{-1}$ but this band is strongly disturbed by vibrations of a different symmetry). In order to analyze this band, we need to take into account the following problems: (i) we cannot see aromatic rings in the spectra separately; (ii) we do not observe absorption bands for certain configuration of the aromatic rings; and (iii) we do not know the "torsional angles" between the aromatic rings.

For example it is known (Tashiro et al. [38]) that biphenyl and its derivatives exhibit the Raman band at 410-420 $\mathrm{cm}^{-1}$ if the biphenyl rings are twisted. However, this band is not observed when biphenyl rings are coplanar. Temperature de- 

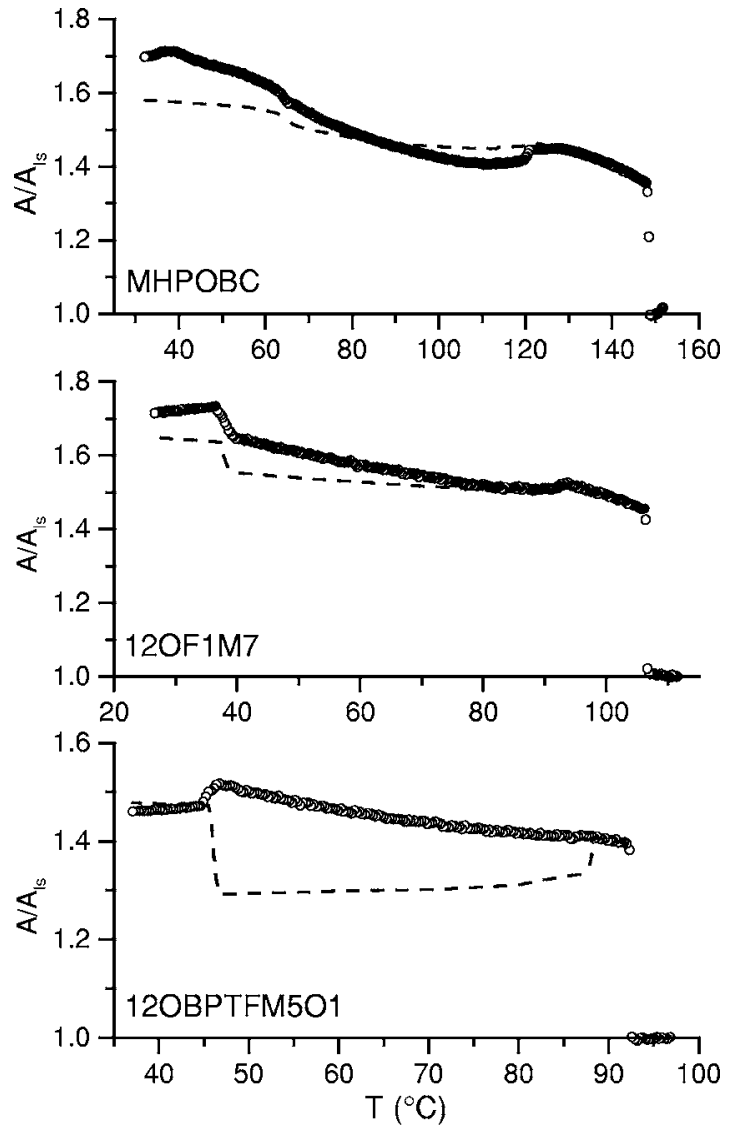

FIG. 9. Absorbances of the phenyl ring out-of-plane bands $\left(760 \mathrm{~cm}^{-1}\right)$ for MHPOBC, 12OF1M7, and 12OBPTFM5O1 vs temperature. Points-experimental values, dashed lines-a numerical simulation of the absorbance in the case of uniform distribution of the transition dipole moments around the axis of the director (i.e., the isotropic distribution of angles $\psi$, see Fig. 1); details of the simulation are given in Fig. 10 and in the text.

pendence of the intensity of this band for MHPOBC (Kim et al. [32]) suggests that biphenyl parts of these molecules are twisted in the mesophase by some angle, and the angle on the average decreases with decreasing temperature. Therefore, the absorbance of the out-of-plane bands contains information about the orientation of the phenyl rings as well as about their possible flattening at lower temperatures. Temperature dependencies of the absorbance of the band $760 \mathrm{~cm}^{-1}$ for the three antiferroelectric compounds are given in Fig. 9. All three dependencies show a similar tendency of increase with decreasing temperature and are only slightly, if at all, affected by an appearance of the molecular tilt. Dashed lines in the plots are simple numerical simulations of the expected absorbance in a hypothetical case of uniform distribution of transition dipole moments around the axis of the director. In order to perform the simulation, a sample set of 200 unit vectors, uniformly distributed on a circle, has been considered (Fig. 10). For the $\mathrm{Sm} A^{*}$ phase, the circle was assumed to be perpendicular to the IR beam (as vectors of the transition dipole moments for the out-of-plane vibration, which are normal to the phenyl ring planes). In lower temperature phases, the circle was tilted by the tilt angle obtained from previous analysis of the phenyl ring stretching

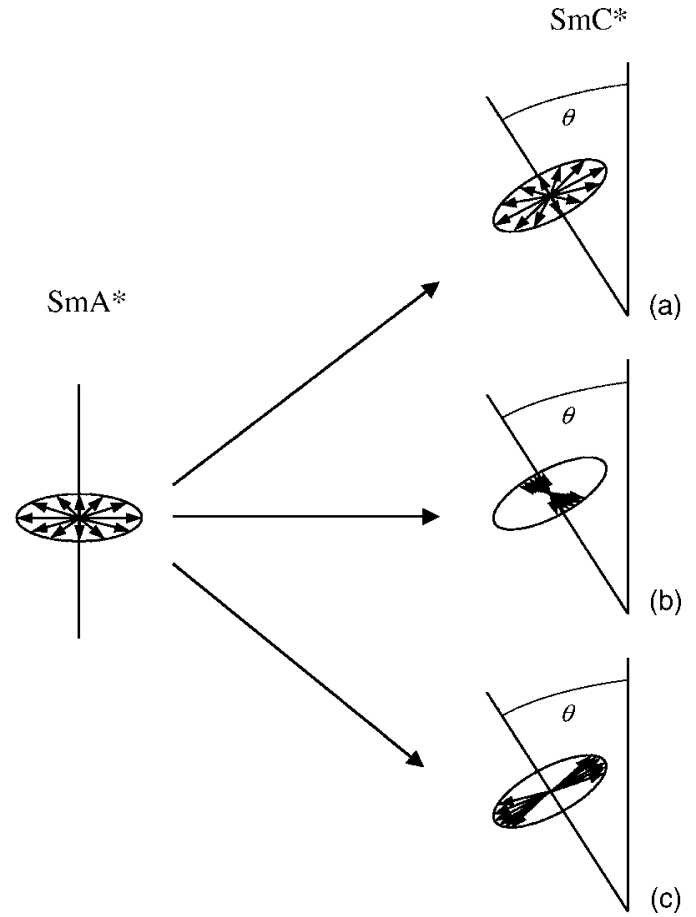

FIG. 10. Schematic diagram of an analysis of the phenyl ring out-of-plane bands. Arrows represent the IR transition dipole moments (in case of the phenyl ring out-of-plane vibration perpendicular to planes of the rings). The vertical line is parallel to the IR beam, $\theta$ is the molecular tilt angle. In the tilted smectic phase, three possible cases are shown: (a) the uniform distribution of the dipoles around the axis of director (isotropic distribution of angles $\psi$ ) is kept, this case is considered in Fig. 9 as dashed lines; (b) the transition dipole moments are on the average oriented rather perpendicular to the molecular tilt plane (i.e., phenyl rings are on the average oriented closer to the molecular tilt plane), then simulated values of the IR absorbance are higher than in the case (a); (c) the transition dipole moments are on the average oriented closer to the molecular tilt plane (i.e., the phenyl rings are on the average oriented rather perpendicular to the molecular tilt plane) and the simulated absorbance is lower than for case (a).

band $\left(1600 \mathrm{~cm}^{-1}\right)$. For each temperature point projections of all the sample vectors were summed up and normalized to unity for the $\operatorname{Sm} A^{*}$ phase range (divided by 200). A power law obtained by fitting experimental points in the range of $\mathrm{Sm} A^{*}$ phase was used to simulate the effect of molecular fluctuations.

The observed deviation from simulated curve shows that the distribution of transition dipole moments is not uniform. The direction of that deviation (into higher values, except in the range $87-127{ }^{\circ} \mathrm{C}$ for MHPOBC, where an inverse tendency is observed) indicates that the transition dipole moments are on the average oriented rather perpendicular to the molecular tilt plane (it means that phenyl rings are oriented on the average close to the molecular tilt plane). What especially is found to be interesting is that the departure from simulated values are the largest for 12OBPTFM5O1, for which the experimentally obtained values of the order parameter $S$ are the lowest. This suggests that the relatively small order of the long molecular axes for this compound 
(and, anyway, not very much higher for 12OF1M7) is compensated by the higher order of the short molecular axes (i.e., higher molecular biaxiality), which results in relatively high values of the spontaneous polarization for this compound $\left(194 \mathrm{nC} \cdot \mathrm{cm}^{-2}\right.$ [39]). This tendency may turn out to be a common feature for materials, for which the first order $\mathrm{Sm} A^{*}-\mathrm{Sm} C^{*}$ transition is observed. Recently Gleeson et al. [40] proposed a model, which takes into account the molecular biaxiality to explain different temperature dependencies of tilt and spontaneous polarization for similar compounds. This seems to be in good qualitative agreement with our data.

\section{CONCLUSIONS}

Infrared spectroscopic results on the apparent order parameter obtained for a group of ferroelectric and antiferroelectric compounds in homeotropic geometry show remarkable consistency, with results of the smectic layer spacing obtained using x-ray diffraction. This is due to the fact that a variation in the apparent order parameter with temperature is largely driven by the tilt, which in turn gives rise to a dependence of the layer thickness on temperature. An almost perfect correspondence between the results using IR spectroscopy and x-ray diffraction is obtained. However, there is a discrepancy between the results of the optical tilt and those obtained using x-ray diffraction. The correspondence of our results with those obtained using $\mathrm{x}$-ray diffraction suggests that the reasons, advanced previously in the literature, of the optical tilt angle being greater than that deduced using x-ray diffraction, need to be reconsidered.

A qualitative analysis of the out-of-plane vibrations of the phenyl rings shows that these rings have a tendency to orient closer to the molecular tilt plane. This tendency is especially strong for molecules, which exhibit a rather low order parameter of the long molecular axes. This observation confirms that the molecular biaxiality plays an important role in the creation of the spontaneous polarization in such a class of AFLCs.

Additional valuable information obtained from a single experiment proves that the given technique may be extremely useful in the investigations of a range of the molecular properties of liquid crystals.

\section{ACKNOWLEDGMENTS}

The work has been funded by the EU project SAMPA Network. We also thank SFI for Grant Nos. 02/W/I02 and 02/IN.1/I031 for funding the visits of Professor A. Fukuda and Professor A. Kocot, respectively, to Trinity College, Dublin, where the work was carried out.
[1] M. Zgonik, M. Rey-Lafon, C. Destrade, C. Leon, and H. T. Nguyen, J. Phys. (France) 51, 2015 (1990).

[2] A. Kocot, G. Kruk, R. Wrzalik, and J. K. Vij, Liq. Cryst. 12, 1005 (1992).

[3] K. H. Kim, K. Ishikawa, H. Takezoe, and A. Fukuda, Phys. Rev. E 51, 2166 (1995).

[4] A. Kocot, J. K. Vij, and T. S. Perova, Adv. Chem. Phys. 113, 203 (2000).

[5] W. G. Jang, C. S. Park, K. H. Kim, M. A. Glaser, and N. A. Clark, Phys. Rev. E 62, 5027 (2000).

[6] W. G. Jang and K. H. Kim, J. Korean Phys. Soc. 38, 4 (2001).

[7] W. G. Jang, J. Korean Phys. Soc. 38, 346 (2001).

[8] W. G. Jang, M. A. Glaser, C. S. Park, K. H. Kim, Y. Lansac, and N. A. Clark, Phys. Rev. E 64, 051712 (2001).

[9] K. Merkel, R. Wrzalik, and A. Kocot, J. Mol. Struct. 563-564, 477 (2001).

[10] R. Wrzalik, K. Merkel, A. Kocot, and B. Ciepłak, J. Chem. Phys. 117, 4889 (2002).

[11] J. Matsushima Y. Takanishi, K. Ishikawa, H. Takezoe, A. Fukuda, C. S. Park, W. G. Jang, K. H. Kim, J. E. MacLennan, and M. A. Glazer, N. A. Clark and K. Takahashi, Liq. Cryst. 29, 27 (2002).

[12] M. D. Ossowska-Chruściel, R. Korlacki, A. Kocot, R. Wrzalik, J. Chruściel, and S. Zalewski, Phys. Rev. E 70, 041705 (2004).

[13] A. Fukuda, J. K. Vij, R. Korlacki, A. Kocot, B. Jin, and Y. Takanishi, Mol. Cryst. Liq. Cryst. 437, 1513 (2005).

[14] D. Dunmur and K. Toriyama, Handbook of Liquid Crystals, edited by D. Demus, J. W. Goodby, G. W. Gray, H. Spiess, and V. Vill (Wiley-VCH, Weinheim, 1998), Vol. 1, p. 189.
[15] K. Merkel, A. Kocot, J. K. Vij, G. H. Mehl, and T. Meyer, J. Chem. Phys. 121, 5012 (2004).

[16] V. P. Panov, N. M. Shtykov, A. Fukuda, J. K. Vij, Y. Suzuki, R. A. Lewis, M. Hird, and J. W. Goodby, Phys. Rev. E 69, 060701(R) (2004).

[17] C. Bahr and G. Heppke, Mol. Cryst. Liq. Cryst. 148, 29 (1987).

[18] C.-D. Poon and B. M. Fung, Liq. Cryst. 5, 1159 (1989).

[19] T. Dollase and B. M. Fung, Liq. Cryst. 21, 915 (1996).

[20] A. D. L. Chandani, Y. Ouchi, H. Takezoe, A. Fukuda, K. Terashima, K. Furukawa, and A. Kishi, Jpn. J. Appl. Phys., Part 2 28, L1261 (1989).

[21] Yu. P. Panarin, O. Kalinovskaya, J. K. Vij, and J. W. Goodby, Phys. Rev. E 55, 4345 (1997).

[22] A. A. Sigarev, J. K. Vij, Yu. P. Panarin, and J. W. Goodby, Phys. Rev. E 62, 2269 (2000).

[23] A. A. Sigarev, J. K. Vij, R. A. Lewis, M. Hird, and J. W. Goodby, Phys. Rev. E 68, 031707 (2003).

[24] M. J. Frisch et al., GAUSSIAN 98W (Revision A.11), Gaussian, Inc., Pittsburgh, PA, 1998.

[25] P. Pulay, G. Fogarasi, G. Pongor, J. E. Boggs, and A. Vargha, J. Am. Chem. Soc. 105, 7037 (1983).

[26] G. Rauhut and P. Pulay, J. Phys. Chem. 99, 3093 (1995).

[27] J. Baker, A. A. Jarzecki, and P. Pulay, J. Phys. Chem. A 102, 1412 (1998).

[28] S. A. Katsyuba, J. Grunenberg, and R. Schmutzler, J. Mol. Struct. 559, 315 (2001).

[29] K. Miyachi, J. Matsushima, Y. Takanishi, K. Ishikawa, H. Takezoe, and A. Fukuda, Phys. Rev. E 52, R2153 (1995). 
[30] F. Hide, N. A. Clark, K. Nito, A. Yasuda, and D. M. Walba, Phys. Rev. Lett. 75, 2344 (1995).

[31] B. Jin, Z. Ling, Y. Takanishi, K. Ishikawa, H. Takezoe, A. Fukuda, M. Kakimoto, and T. Kitazume, Phys. Rev. E 53, (R) 04295 (1996).

[32] K. H. Kim, Y. Takanishi, K. Ishikawa, H. Takezoe, and A. Fukuda, Liq. Cryst. 16, 185 (1994).

[33] M. Neundorf, Y. Takanishi, A. Fukuda, S. Saito, K. Murashiro, T. Inkunai, and D. Demus, J. Mater. Chem. 5, 2221 (1995).

[34] Y. Takanishi, K. Miyachi, S. Yoshida, B. Jin, H. Yin, K. Ishikawa, H. Takezoe, and A. Fukuda, J. Mater. Chem. 8, 1133 (1998)
[35] N. Hayashi and T. Kato, Phys. Rev. E 63, 021706 (2001).

[36] L. S. Matkin, H. F. Gleeson, L. J. Baylis, S. J. Watson, N. Bowring, A. Seed, M. Hird, and J. W. Goodby, Appl. Phys. Lett. 77, 340 (2000).

[37] J. T. Mills, H. F. Gleeson, J. W. Goodby, M. Hird, A. Seed, and P. Styring, J. Mater. Chem. 8, 2385 (1998).

[38] K. Tashiro, J. Hou, M. Kobayashi, and T. Inoue, J. Am. Chem. Soc. 112, 8273 (1990).

[39] V. Görtz (private communication).

[40] H. F. Gleeson, Y. Wang, S. Watson, D. Sahagun-Sanchez, J. W. Goodby, M. Hird, A. Petrenko, and M. A. Osipov, J. Mater. Chem. 14, 1480 (2004). 